\title{
Expansion of Cell Range with Geometric Information of Pico-Cell for Maximum Sum Rate in Heterogeneous Networks
}

\author{
Taesung JUNG ${ }^{1}$, Iickho SONG ${ }^{1}$, Seungwon $L E E^{1}$, \\ Seungjae JUNG ${ }^{1}$, Seokho YOON ${ }^{2}$, Joonhyuk KANG ${ }^{1}$ \\ ${ }^{1}$ School of Electrical Engineering, Korea Advanced Institute of Science and Technology, Daejeon 34141, Korea \\ ${ }^{2}$ College of Information and Communication Engineering, Sungkyunkwan University, Suwon 16419, Korea
}

10hdg@naver.com, i.song@iieee.org, kkori21@gmail.com, sjjung513@kaist.ac.kr, syoon@ skku.edu, jhkang@kaist.edu

Submitted October 3, 2017 / Accepted January 24, 2018

\begin{abstract}
In this paper, taking the positions of pico-cell base stations (PBSs) into consideration, a scheme of cell range expansion (CRE) for maximum sum rate is addressed in heterogeneous multi-input multi-output multi-user wireless networks. The optimal CRE bias obtained numerically by the proposed CRE scheme with inter-cell interference coordination (ICIC) allows us to maximize the sum rate while successfully maintaining the load balance between the macrocell base station and PBSs. Numerical results confirm that the proposed CRE scheme with ICIC can provide higher sum rate than conventional schemes and balanced load.
\end{abstract}

\section{Keywords}

Cell range expansion (CRE), heterogeneous network, interference coordination, multi-input multi-output multi-user (MIMO-MU) system

\section{Introduction}

Consisting of macro-cells overlaid by smaller cells such as micro-cells, femto-cells, and pico-cells, heterogeneous networks have been proposed as a breakthrough for an efficient deployment of base stations (BSs) in space and spectrum [1], [2]. One of the advantages of heterogeneous networks is the reduced burden of macro-cell base stations (MBSs) with the smaller cells incorporated, leading to a highly efficient network design. On the other hand, since various types of BSs are incorporated, the network planning, resource allocation design, and interference management may become more complicated. Apparently, various aspects of the heterogeneous networks have been investigated including cell assignment strategy [3], [4], power control schemes [5], coverage area [6-8], stochastic geometry models [9], [10], and cell range expansion [11].
Unlike in homogeneous networks, in which the BS that provides the highest downlink signal power is selected as the serving BS, the variety of level of power for various BSs should be taken into account in heterogeneous networks. Specifically, since the transmission power of a picocell BS (PBS) is lower than that of the MBS, most users would choose the MBS instead of a PBS as the serving BS, which naturally leads to load unbalance among the BSs. Addressing this problem, the scheme proposed in [11] expands the cell range by imposing a bias to the reference signal received power (RSRP) from the PBS, consequently making some macro-cell users be offloaded to a pico-cell.

Although the cell range expansion (CRE) improves the performance (i.e., overall sum rate) of the heterogeneous network, CRE users (that is, users offloaded to pico-cells) suffer from high inter-cell interference (ICI) from the MBS. To avoid the decrease in the overall sum rate of the system resulting from the high ICI, the MBS normally employs inter-cell interference coordination (ICIC) with the CRE [11], [12]. Among the typical research on CRE are capacity and fairness analysis [13], resource partitioning [14], adaptive CRE bias control [15], cell association [16], and offloading performance [17]. In addition, an algorithm that allows each user to determine CRE bias value to lower the number of outage users is proposed in [18]. The CRE method in [19] optimizes the parameters by evaluating the average throughput of the cell-edge users as well as the other users. The optimal CRE bias value for various density of small cell clusters in the coverage area of the macro-cell has been analyzed in [20]. Adaptive CRE schemes have also been proposed taking into account varying load among the MBS and PBSs [21] and overall capacity [22].

In this paper, we propose a CRE scheme in which the positions of PBSs are taken into account for the determination of the CRE bias, which have not been considered in other research of the CRE. In the proposed CRE scheme, the CRE bias, as a function of the PBS location, is determined to max- 
imize the overall sum rate of the system. Through simulation results, we have shown that the proposed CRE scheme with ICIC has better overall performance and more balanced load among BSs than the conventional schemes.

The rest of this paper is organized as follows. After a description of the system model assumed in this paper, the problem is formulated in Sec. 2. Section 3 provides some results from numerical simulations and performance analysis. Section 4 concludes this paper.

\section{System Model and Problem Formu- lation}

Consider the downlink of a heterogeneous network in a multi-input multi-output multi-user (MIMO-MU) system. We assume that the heterogeneous network is composed of one macro-cell overlaid by $C_{\mathrm{P}}$ pico-cells, where the MBS and each of the $C_{\mathrm{P}}$ PBSs are equipped with $N_{\mathrm{M}}$ and $N_{\mathrm{P}}$ antennas, respectively, with $N_{\mathrm{M}}>N_{\mathrm{P}}$. It is also assumed that the users, each equipped with a single antenna, are uniformly distributed over the cell.

Let the transmit power of the MBS and each of the $C_{\mathrm{P}}$ PBSs be $P_{\mathrm{M}}$ and $P_{\mathrm{P}}$, respectively, with $P_{\mathrm{M}}>P_{\mathrm{P}}$, and denote by $\alpha_{\mathrm{M}}$ and $\alpha_{\mathrm{P}}$ the pathloss exponents of the macro-cells and pico-cells, respectively. Assuming simplified pathloss model, the RSRP from the MBS and a PBS can be expressed as $P_{\mathrm{M}} \tilde{d}_{\mathrm{M}}^{-\alpha_{\mathrm{M}}}$ and $P_{\mathrm{P}} \tilde{d}_{\mathrm{P}}^{-\alpha_{\mathrm{P}}}$, respectively, where $\tilde{d}_{\mathrm{M}}$ and $\tilde{d}_{\mathrm{P}}$ are the distances between a user and the MBS and PBS, respectively.

\subsection{Expansion of Pico-Cell Range in Heteroge- neous Network}

We will denote the BS of the $k$ th cell and the $i$ th user in the $j$ th cell by BS- $k$ and User- $(i, j)$, respectively: Here, without loss of generality, we assume that the zero-th cell is the macro-cell and the first, second, $\ldots, C_{\mathrm{P}}$-th cells are pico-cells. In addition, by Situations $\mathrm{A}$ and $\mathrm{B}$, we denote the cases in which the MBS does not and does, respectively, incorporate ICIC via zero-forcing for users in pico-cells: In any of the two situations, no PBS performs ICIC.

The serving BS, to which a user reports the channel state information (CSI) and through which the user transmits and receives the information signal, of a user is determined by comparing the RSRP from BSs. Specifically, without CRE, the serving BS of user $i$ will be BS- $k(i)$ when

$$
k(i)=\underset{j}{\arg \max } \mathrm{RSRP}_{i, j}
$$

is satisfied, where

$$
\operatorname{RSRP}_{i, j}= \begin{cases}P_{\mathrm{P}} \tilde{d}_{i, j}^{-\alpha_{\mathrm{P}},}, & \text { if BS- } j \text { is a PBS, } \\ P_{\mathrm{M}} \tilde{d}_{i, j}^{-\alpha_{\mathrm{M}}}, & \text { if BS- } j \text { is the MBS }\end{cases}
$$

denotes the RSRP of the $i$ th user from BS- $j$ with $\tilde{d}_{i, j}$ the distance between the $i$ th user and BS- $j$.
Now, consider a CRE scheme with which the serving BS of the $i$-th user is determined by

$$
k(i)=\underset{j}{\arg \max }\left\{b_{j} \operatorname{RSRP}_{i, j}\right\},
$$

where

$$
b_{j}= \begin{cases}b, & \text { if } \mathrm{BS} j \text { is a PBS, } \\ 1, & \text { if } \mathrm{BS} j \text { is the MBS }\end{cases}
$$

is the bias with $b \geq 1$. Equation (3) basically implies that we have now expanded the cell ranges of pico-cells. Clearly, when the ranges of pico-cells are expanded, some users in the macro-cell will be offloaded to a pico-cell.

Once the serving BS for every user is determined, the received signals $y_{i, 0, S}$ and $\left\{y_{i, j, S}\right\}_{j=1}^{C_{\mathrm{P}}}$ of users in the macro- and pico-cells, respectively, under Situation $S$ can be expressed as

$$
\begin{aligned}
& y_{i, 0, S}=\sqrt{\frac{P_{\mathrm{M}}}{U_{\mathrm{M}}} d_{i, 0,0}^{-\alpha_{\mathrm{M}}}} \\
& \times\{\boldsymbol{h}_{i, 0,0}^{*} \boldsymbol{f}_{i, 0, S} x_{i, 0}+\underbrace{\sum_{m=1, m \neq i}^{U_{\mathrm{M}}} \boldsymbol{h}_{i, 0,0}^{*} \boldsymbol{f}_{m, 0, S} x_{m, 0}}_{\text {intra-cell interference }}\} \\
& +\underbrace{\sum_{k=1}^{C_{\mathrm{P}}} \sum_{p=1}^{U_{P_{k}}} \sqrt{\frac{P_{\mathrm{P}}}{U_{P_{k}}} d_{i, 0, k}^{-\alpha_{\mathrm{P}}} \boldsymbol{h}_{i, 0, k}^{*} \boldsymbol{f}_{p, k, S} x_{p, k}}+z_{i, 0}}_{\text {inter-cell interference }}
\end{aligned}
$$

and

$$
\begin{aligned}
& y_{i, j, S}=\sqrt{\frac{P_{\mathrm{P}}}{U_{P_{j}}} d_{i, j, j}^{-\alpha_{\mathrm{P}}}} \\
& \times\{\boldsymbol{h}_{i, j, j}^{*} \boldsymbol{f}_{i, j, S} x_{i, j}+\underbrace{\sum_{\text {intra-cell interference }}^{U_{\mathrm{P}_{j}}} \boldsymbol{h}_{i, j, j}^{*} \boldsymbol{f}_{p, j, S} x_{p, j}}_{p=1, p \neq i}\} \\
& +\sum_{m=1}^{U_{\mathrm{M}}} \sqrt{\frac{P_{\mathrm{M}}}{U_{\mathrm{M}}} d_{i, j, 0}^{-\alpha_{\mathrm{M}}} \boldsymbol{h}_{i, j, 0}^{*} \boldsymbol{f}_{m, 0, S} x_{m, 0}} \\
& +\sum_{k=1, k \neq j}^{C_{\mathrm{P}}} \sum_{\text {inter-cell interference }}^{U_{\mathrm{P} k}} \sqrt{\frac{P_{\mathrm{P}}}{U_{\mathrm{P} k}} d_{i, j, k}^{-\alpha_{\mathrm{M}}} \boldsymbol{h}_{i, j, k}^{*} \boldsymbol{f}_{p, k, S} x_{p, k}}
\end{aligned}
$$

for $j=1,2, \ldots, C_{\mathrm{P}}$, where $S \in\{\mathrm{A}, \mathrm{B}\}$ is the set of the two situations we consider; $U_{\mathrm{M}}$ and $U_{\mathrm{P}_{k}}$ for $k=1,2, \ldots, C_{\mathrm{P}}$ are the numbers of users in the macro- and $k$-th pico-cells, respectively; $d_{i, j, k}$ is the distance between User- $(i, j)$ and BS- $k$; $\boldsymbol{f}_{i, j, S}$ is the precoder of User- $(i, j)$ under Situation $S ; \boldsymbol{h}_{i, j, k}$ is the channel constant between User- $(i, j)$ and BS- $k$ and is modeled as a zero-mean uncorrelated fading with unit variance 
and Rayleigh-distributed envelope; $x_{i, j}$ is the desired signal for User- $(i, j)$ with power constraint $E\left[\left|x_{i, j}\right|^{2}\right]=1$; and $z_{i, j}$ is the complex Gaussian noise for User- $(i, j)$. In passing, let us note that the precoder $\boldsymbol{f}_{i, j, S}$ is of size $N_{\mathrm{M}} \times 1$ when $j=0$ and $N_{\mathrm{P}} \times 1$ when $j=1,2, \ldots, C_{\mathrm{P}}$ with $\left\|\boldsymbol{f}_{i, j, S}\right\|^{2}=1$ and that the channel constant $\boldsymbol{h}_{i, j, k}$ is of size $N_{\mathrm{M}} \times 1$ for $k=0$ and $N_{\mathrm{P}} \times 1$ for $k=1,2, \ldots, C_{\mathrm{P}}$. It should be noted that the received signals $y_{i, 0, S}$ and $\left\{y_{i, j, S}\right\}_{j=1}^{C_{\mathrm{P}}}$ are functions of the bias $b$ although we have not shown the dependence explicitly for a brevity reason.

Clearly, macro-cell users would suffer intra-cell interference from the MBS and ICI from the PBSs. Similarly, pico-cell users would suffer intra-cell interference from the serving PBS and ICI from the MBS and other PBSs. Now, since the transmit power of the MBS is normally much higher than that of the PBSs, pico-cell users, especially the CRE users, suffer high ICI from the MBS. To alleviate the influence of such interference, we employ precoders at the BSs. Specifically, the precoders used in the BSs can be expressed as

$$
\boldsymbol{f}_{i, 0, \mathrm{~A}}=\frac{\boldsymbol{h}_{i, 0,0}}{\left\|\boldsymbol{h}_{i, 0,0}\right\|}
$$

for $i=1,2, \ldots, U_{\mathrm{M}}$ and

$$
\boldsymbol{f}_{i, j, \mathrm{~A}}=\frac{\boldsymbol{h}_{i, j, j}}{\left\|\boldsymbol{h}_{i, j, j}\right\|}
$$

for $i=1,2, \ldots, U_{\mathrm{P}_{j}}$ and $j=1,2, \ldots, C_{\mathrm{P}}$ under Situation A, and

$$
\boldsymbol{f}_{i, 0, \mathrm{~B}}=\frac{\left\{\boldsymbol{I}_{N_{\mathrm{M}}}-\boldsymbol{H}\left(\boldsymbol{H}^{*} \boldsymbol{H}\right)^{-1} \boldsymbol{H}^{*}\right\} \boldsymbol{h}_{i, 0,0}}{\left\|\left\{\boldsymbol{I}_{N_{\mathrm{M}}}-\boldsymbol{H}\left(\boldsymbol{H}^{*} \boldsymbol{H}\right)^{-1} \boldsymbol{H}^{*}\right\} \boldsymbol{h}_{i, 0,0}\right\|}
$$

for $i=1,2, \ldots, U_{\mathrm{M}}$ and

$$
f_{i, j, \mathrm{~B}}=f_{i, j, \mathrm{~A}}
$$

for $i=1,2, \ldots, U_{\mathrm{P}_{j}}$ and $j=1,2, \ldots, C_{\mathrm{P}}$ under Situation B. Here, \|\|$, \boldsymbol{I}_{n}$, and the superscript $*$ denote the Euclidean norm, $n \times n$ identity matrix, and complex conjugate transpose, respectively, and the matrix

$$
\boldsymbol{H}=\left[\begin{array}{llll}
\boldsymbol{H}_{1} & \boldsymbol{H}_{2} & \ldots & \boldsymbol{H}_{C_{\mathrm{P}}}
\end{array}\right]
$$

of the channel constants is of size $N_{\mathrm{M}} \times U_{\mathrm{P}}$ with $\boldsymbol{H}_{j}=\left[\begin{array}{llll}\boldsymbol{h}_{1, j, 0} & \boldsymbol{h}_{2, j, 0} & \ldots & \boldsymbol{h}_{U_{\mathrm{P}_{j}}, j, 0}\end{array}\right]$ and $U_{\mathrm{P}}=\sum_{k=1}^{C_{\mathrm{P}}} U_{\mathrm{P}_{k}}$ the total number of users in the $C_{\mathrm{P}}$ pico-cells.

Equations (7) and (8) indicate that the MBS and PBSs both employ eigen-beamforming for their own users with no ICIC in Situation A. In Situation B on the other hand, as implied in (9) and (10), the MBS employs zero-forcing in order to reduce the interference toward the users in the picocells [23] while the PBSs still exploit eigen-beamforming. Here, the MBS normally exploits many antennas and high transmission power and thus the performance degradation of the macro-cell users due to the ICIC via zero-forcing would be, if not negligible, small while the ICIC would allow significant performance enhancement for pico-cell users. On the other hand, as the PBS has less antennas and lower transmission power, performance degradation of the pico-cell users would be severe if the PBS performed ICIC via zero-forcing. This is why only MBS performs ICIC and the PBS exploits eigen-beamforming regardless of the situation.

The signal to interference plus noise ratio (SINR) can now be expressed as

$$
\mathrm{SINR}_{i, 0, S}=\frac{P_{\mathrm{M}} d_{i, 0,0}^{-\alpha_{\mathrm{M}}}\left|\boldsymbol{h}_{i, 0,0}^{*} \boldsymbol{f}_{i, 0, S}\right|^{2}}{U_{\mathrm{M}}\left(N_{0} W+I_{i, 0, S}\right)}
$$

for macro-cell users and

$$
\operatorname{SINR}_{i, j, S}=\frac{P_{\mathrm{P}} d_{i, j, j}^{-\alpha_{\mathrm{P}}}\left|\boldsymbol{h}_{i, j, j}^{*} \boldsymbol{f}_{i, j, S}\right|^{2}}{U_{\mathrm{P}_{j}}\left(N_{0} W+I_{i, j, S}\right)}
$$

for pico-cell users, where $W$ is the system bandwidth, $N_{0}$ is the noise power per unit bandwidth, and

$$
\begin{aligned}
& I_{i, 0, S}=\sum_{m=1, m \neq i}^{U_{\mathrm{M}}} \frac{P_{\mathrm{M}}}{U_{\mathrm{M}}} d_{i, 0,0}^{-\alpha_{\mathrm{M}}}\left|\boldsymbol{h}_{i, 0,0}^{*} \boldsymbol{f}_{m, 0, S}\right|^{2} \\
& +\sum_{k=1}^{C_{\mathrm{P}}} \sum_{p=1}^{U_{\mathrm{P} k}} \frac{P_{\mathrm{P}}}{U_{\mathrm{P} k}} d_{i, 0, k}^{-\alpha_{\mathrm{P}}}\left|\boldsymbol{h}_{i, 0, k}^{*} \boldsymbol{f}_{p, k, S}\right|^{2}
\end{aligned}
$$

and

$$
\begin{aligned}
& I_{i, j, S}=\sum_{m=1}^{U_{\mathrm{M}}} \frac{P_{\mathrm{M}}}{U_{\mathrm{M}}} d_{i, j, 0}^{-\alpha_{\mathrm{M}}}\left|\boldsymbol{h}_{i, j, 0}^{*} \boldsymbol{f}_{m, 0, S}\right|^{2} \\
& +\sum_{p=1, p \neq i}^{U_{\mathrm{P}}} \frac{P_{\mathrm{P}}}{U_{\mathrm{P}}} d_{i, j, j}^{-\alpha_{\mathrm{P}}}\left|\boldsymbol{h}_{i, j, j}^{*} \boldsymbol{f}_{p, j, S}\right|^{2} \\
& +\sum_{k=1, k \neq j}^{C_{\mathrm{P}}} \sum_{p=1}^{U_{\mathrm{P} k}} \frac{P_{\mathrm{P}}}{U_{\mathrm{P} k}} d_{i, j, k}^{-\alpha_{\mathrm{P}}}\left|\boldsymbol{h}_{i, j, k}^{*} \boldsymbol{f}_{p, k, S}\right|^{2}
\end{aligned}
$$

denote the total interference in the macro- and pico-cells, respectively. When the MBS incorporates the ICIC, the ICI term (that is, the first term in the right-hand side) of (15) will vanish. Note that, when we have only one PBS, the last term of (15) will be zero.

Eventually, we try to find the optimal CRE bias value

$$
b^{*}=\underset{b}{\arg \max } \sum_{j} \sum_{i} \text { Rate }_{i, j, S}
$$

for which the sum rate of overall system is maximized, where

$$
\text { Rate }_{i, j, S}=W \log _{2}\left(1+\operatorname{SINR}_{i, j, S}\right)
$$

is the achievable rate for User- $(i, j)$ under Situation $S$. As we shall see shortly, the overall sum rate first increases and then decreases as the value $b$ of the CRE bias increases. 


\subsection{Two Examples of Heterogeneous Networks}

One PBS (1-PBS) model: The simplest case of heterogeneous networks with one macro-cell and one pico-cell $\left(C_{\mathrm{P}}=1\right)$ is shown in Fig. 1. The two small circles of solid and dash-dot lines indicate the ranges of the pico-cell without and with the CRE, respectively. The solid and dotted arrows indicate desired (information data) signal and ICI, respectively, with the CRE. The user between the two small circles is the CRE user, offloaded to the PBS, and would suffer high ICI from the MBS.

Three PBS (3-PBS) model: Figure 2 shows the heterogeneous network with one macro-cell and three pico-cells. It is easy to see that the ICI will be higher with more PBSs as we have already observed in, for example, (6) and (15).

\section{Numerical Results and Analysis}

Let us now consider some simulation results, for which the simulation parameters in the 1-PBS model are shown in Tab. 1. Here, the values of the transmit power of the BSs and the pathloss exponents of the macro- and pico-cells are adopted from those employed in [24]. It is assumed that the transmit power of a BS is equally distributed to its users: Thus, the power received by each user is determined by the type of its serving BS and the number of users dwelling in the same cell. The maximum value of the CRE bias is set to $12 \mathrm{~dB}$, at which the coverage area of a pico-cell becomes almost the same as that of the macro-cell. For the 3-PBS model, we have in addition assumed the minimum distance between two PBSs to be $100 \mathrm{~m}$.

Since the number of users serviced simultaneously is upper-limited by the number of antennas of the MBS, we assume that the total number of users in the heterogeneous network is 8 for simplicity. When the number of users in a pico-cell reaches the number 4 of antennas of the PBS, no additional macro-cell user will be offloaded even when the pico-cell range is expanded with the CRE. Note that the minimum and maximum distances between BSs are considered to avoid the cases in which the PBS is too close to the
MBS or to the boundary of the macro-cell: In both cases, the coverage area of the PBS would become too small.

Without loss of generality, we assume that the MBS and PBS are located at $(0,0)$ and $\left(x_{\mathrm{P}}, 0\right)$, respectively, on the $x$-axis in the 1-PBS model, with two additional PBSs at $\left(x_{\mathrm{P}} \cos \frac{2 \pi}{3}, \pm x_{\mathrm{P}} \sin \frac{2 \pi}{3}\right)$ in the 3-PBS model on a twodimensional plane.

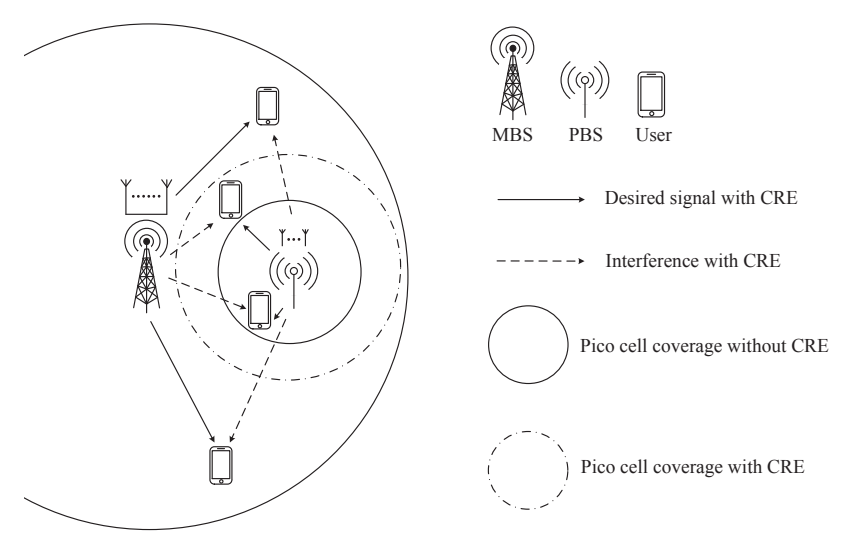

Fig. 1. Heterogeneous network: 1-PBS model.

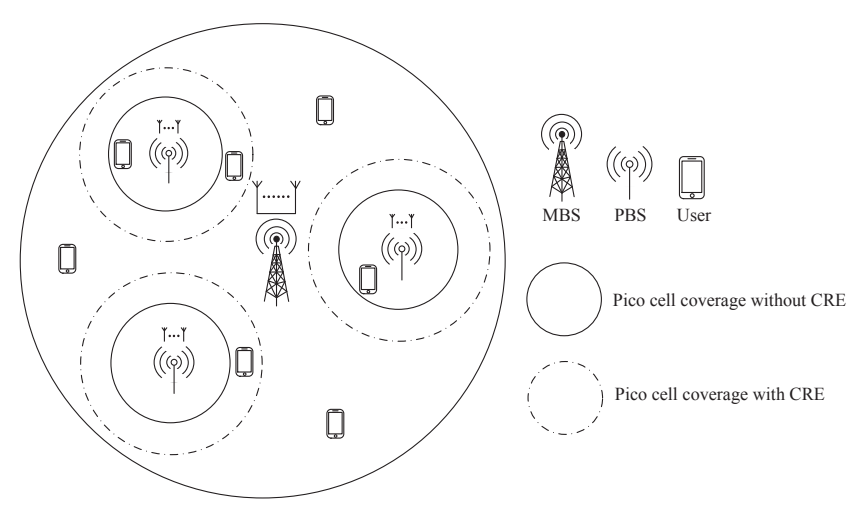

Fig. 2. Heterogeneous network: 3-PBS model.

\begin{tabular}{|c|c|c|}
\hline & Macro-cell & Pico-cell \\
\hline Number of antennas & $N_{\mathrm{M}}=8$ & $N_{\mathrm{P}}=4$ \\
Transmit power & $P_{\mathrm{M}}=46 \mathrm{dBm}$ \\
$P_{\mathrm{M}}=3.76$ & $\alpha_{\mathrm{P}}=3.67$ \\
Pathloss exponent & \multicolumn{2}{|c|}{8} \\
Total number of users & $1000 \mathrm{~m}$ \\
Macro-cell radius & $200 \mathrm{~m}$ \\
Minimum distance between MBS and PBS & $800 \mathrm{~m}$ \\
Maximum distance between MBS and PBS & $0 \sim 12 \mathrm{~dB}$ \\
CRE bias range & $\alpha_{0}=-174 \mathrm{dBm} / \mathrm{Hz}$ \\
Noise power per bandwidth & $W=10 \mathrm{MHz}$ \\
System bandwidth & \multicolumn{2}{|c}{} \\
\hline
\end{tabular}

Tab. 1. Values of parameters used in simulation. 


\subsection{Bias and Sum Rate}

Figures 3 and 4 show the sum rate as a function of the bias of the proposed CRE scheme in the 1-PBS and 3-PBS models, respectively, when the distance between the MBS and PBS is 200, 500, and $800 \mathrm{~m}$. Clearly, it is confirmed that the proposed CRE scheme increases the overall sum rate of the system when the ICIC is applied: Such increase of overall sum rate has been observed, for example, in [25], [26] also. It is observed that, as the PBS is located closer to the MBS, the sum rate varies more with the CRE bias. It should be noted that, at each location of the PBS, there exists an optimal value of the CRE bias maximizing the sum rate and that the optimal value is dependent on the distance between the MBS and PBS.

Figures 5 and 6 show the sum rate with the proposed CRE scheme as a function of the distance between the MBS and PBS at several values of the CRE bias.

It is again observed that the ICIC increases the sum rate at any value of the CRE bias irrespective of the PBS position. It is also observed that the increase of the sum rate with the optimum CRE bias is more considerable with the ICIC.

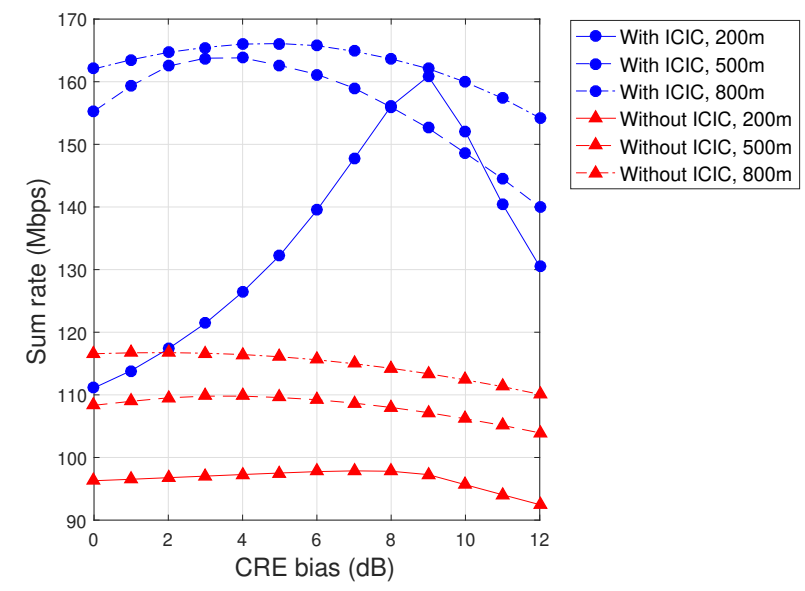

Fig. 3. Sum rate when the distance between the MBS and PBS is 200,500 , and $800 \mathrm{~m}$ in the 1-PBS model.

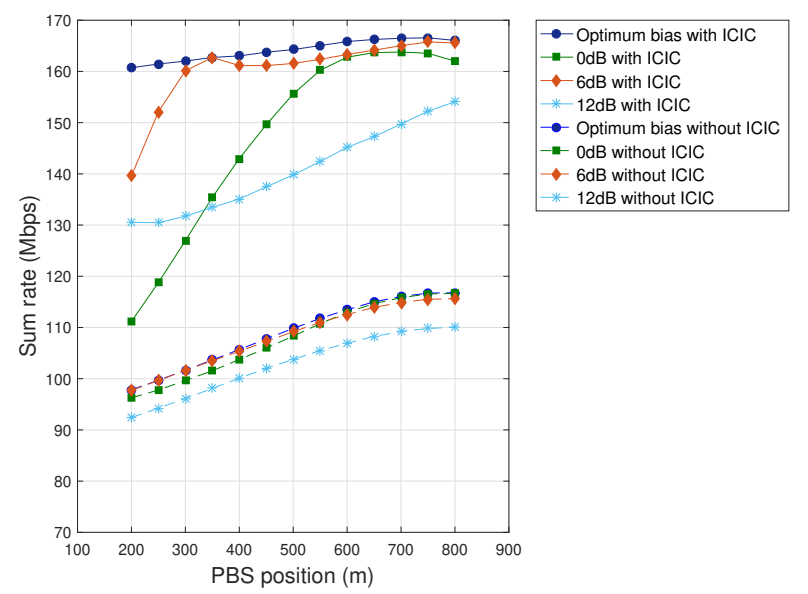

Fig. 5. Sum rate when the distance between the MBS and PBS varies from 200 to $800 \mathrm{~m}$ in the 1-PBS model.
For example, consider the sum rate when the PBS is $200 \mathrm{~m}$ from the MBS in Fig. 5. The sum rate with the CRE bias of $0 \mathrm{~dB}$ is slightly over $95 \mathrm{Mbps}$ and that with the optimum bias is less than $100 \mathrm{Mbps}$, implying that the optimum CRE provides us with an increase of about $5 \mathrm{Mbps}$ in the sum rate. On the other hand, with the ICIC applied, the sum rate with the CRE bias of $0 \mathrm{~dB}$ is around $110 \mathrm{Mbps}$ and that with the optimum bias is around $160 \mathrm{Mbps}$. In essence, the increase in the sum rate with the ICIC is around $50 \mathrm{Mbps}$.

From the comparison of the results in Figs. 5 and 6 , we may conclude that the sum rate in the 3-PBS model is generally higher than that in the 1-PBS model. We believe this is due to the fact that, although the interference would also be increased, more PBSs in a fixed space imply more antennas and higher transmit power in addition to decreased average distance between the user and BS, resulting in increased average received power for users.

Figures 7 and 8 show the optimal CRE bias that induces the maximum sum rate as a function of the distance between the MBS and PBS. The optimal CRE bias tends to decreases when the distance between the MBS and PBS increases. This is due to the increase of the coverage area of the PBS.

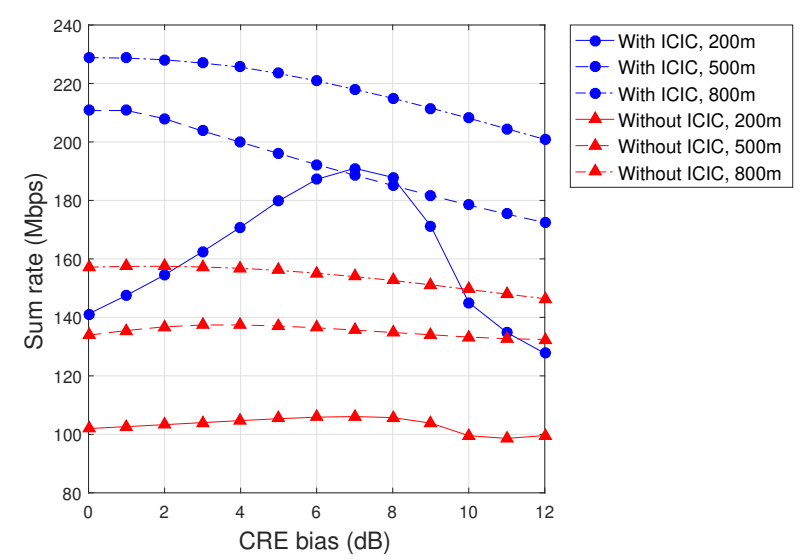

Fig. 4. Sum rate when the distance between the MBS and a PBS is 200,500 , and $800 \mathrm{~m}$ in the 3-PBS model.

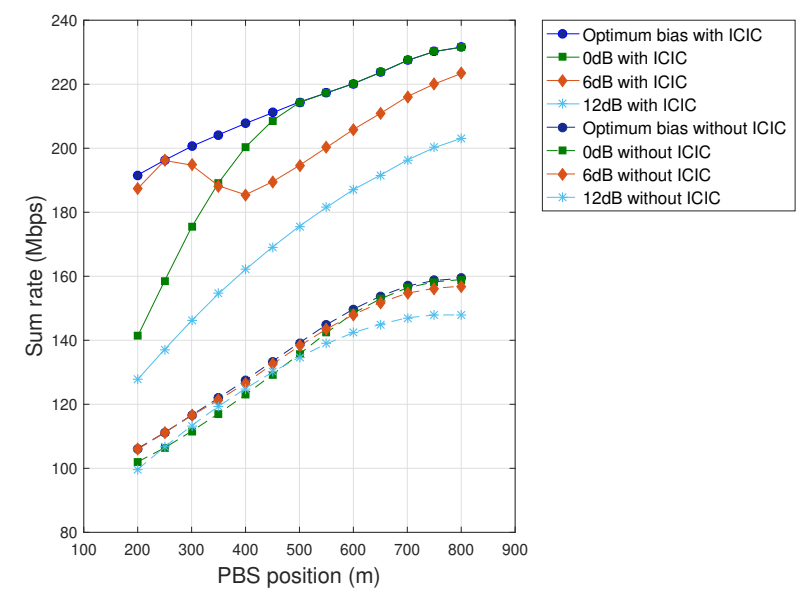

Fig. 6. Sum rate when the distance between the MBS and PBS varies from 200 to $800 \mathrm{~m}$ in the 3-PBS model. 


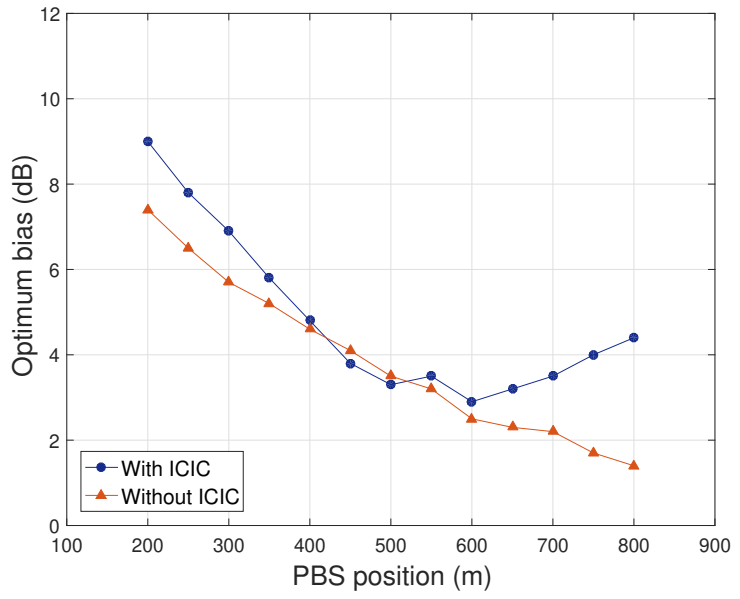

Fig. 7. Optimum CRE bias value versus the distance between the MBS and PBS in the 1-PBS model.

\subsection{Coverage Area}

The boundary of the pico-cell can be expressed as

$$
P_{\mathrm{M}}\left(x^{2}+y^{2}\right)^{-\frac{\alpha_{\mathrm{M}}}{2}}=P_{\mathrm{P}}\left\{\left(x-x_{\mathrm{P}}\right)^{2}+y^{2}\right\}^{-\frac{\alpha_{\mathrm{P}}}{2}},
$$

or equivalently as

$$
\left\{\left(x-x_{\mathrm{P}}\right)^{2}+y^{2}\right\}^{\alpha_{\mathrm{P}}}-\left(\frac{P_{\mathrm{P}}}{P_{\mathrm{M}}}\right)^{2}\left(x^{2}+y^{2}\right)^{\alpha_{\mathrm{M}}}=0,
$$

which can eventually be approximated as an ellipse under the assumption that the coverage area of the macro-cell is unbounded [7]: Some examples are shown in Figs. 9-11 for the 1-PBS model. In these figures, a user located at the positions of black and red dots represents a macro- and a pico-cell users, respectively.

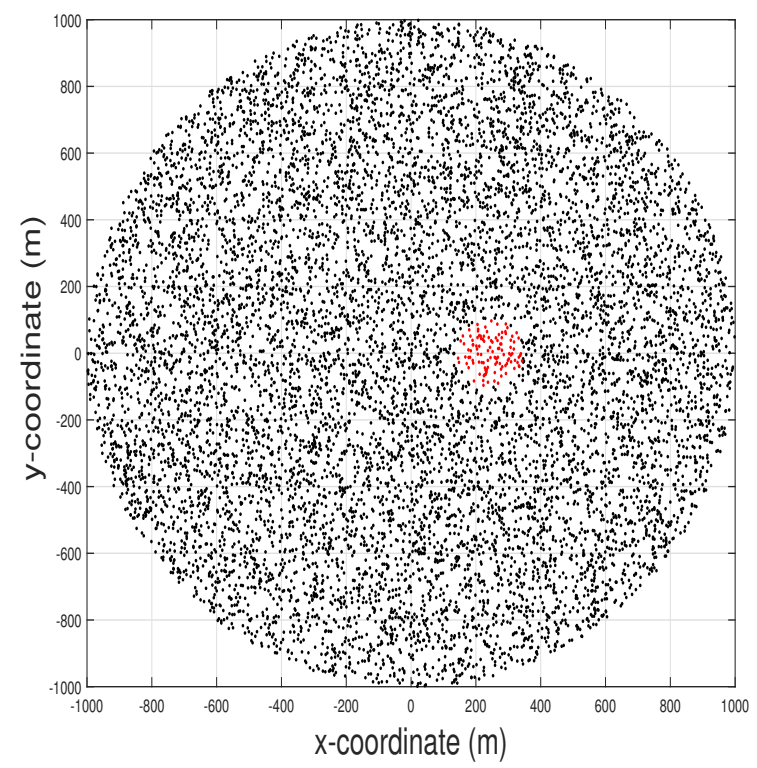

Fig. 9. Coverage areas of the MBS and PBS when the MBS and PBS are located at $(0,0)$ and $(200,0)$, respectively.

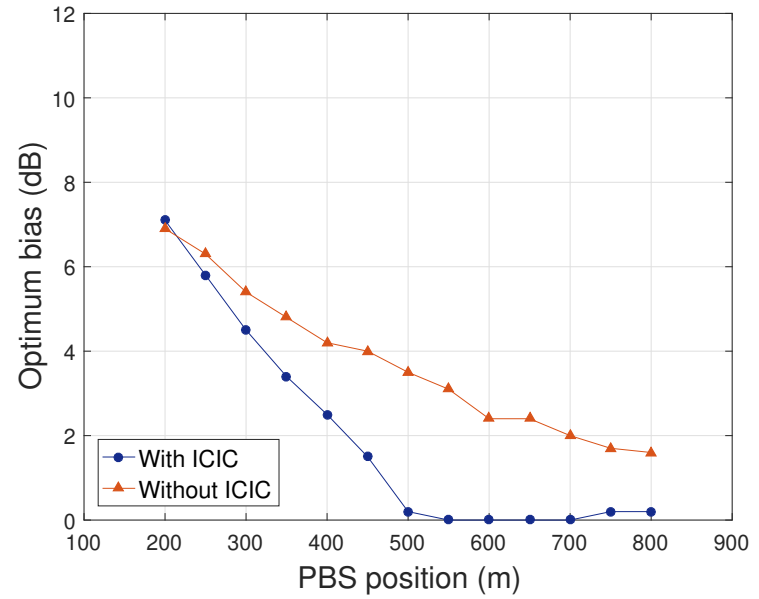

Fig. 8. Optimum CRE bias value versus the distance between the MBS and a PBS in the 3-PBS model.

Figure 12 shows the coverage areas of the PBS without any CRE, with the proposed CRE scheme only, and with the proposed CRE scheme plus ICIC, where 'proposed scheme' denotes the proposed CRE scheme with an optimum bias value. It is observed that the coverage area of the PBS increases with the proposed CRE scheme, and that the ICIC makes the coverage of PBS less dependent on the distance between the PBS and MBS.

Figure 13 shows the ratio of the coverage area with the proposed CRE scheme to that without a CRE scheme in decibel scale. Clearly, when the ICIC is employed with the proposed scheme, the ratio (denoted by blue circles) decreases and then increases slightly after a certain point as the PBS is located farther from the MBS. On the other hand, when the ICIC is not employed with the proposed scheme, the ratio (denoted by red triangles) decreases monotonically. It is interesting to note the resemblance between Figs. 7 and 13.

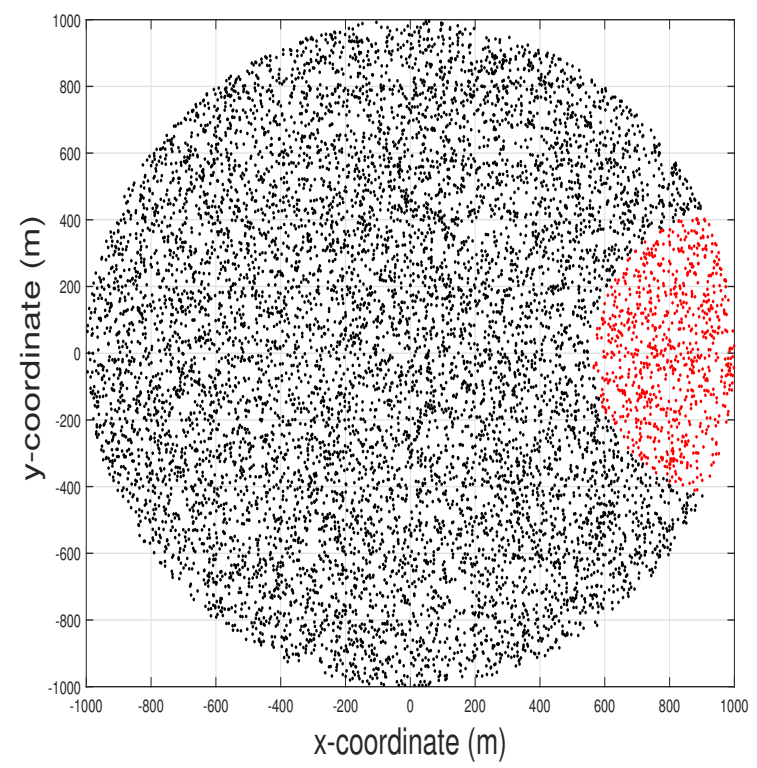

Fig. 10. Coverage areas of the MBS and PBS when the MBS and PBS are located at $(0,0)$ and $(800,0)$, respectively. 


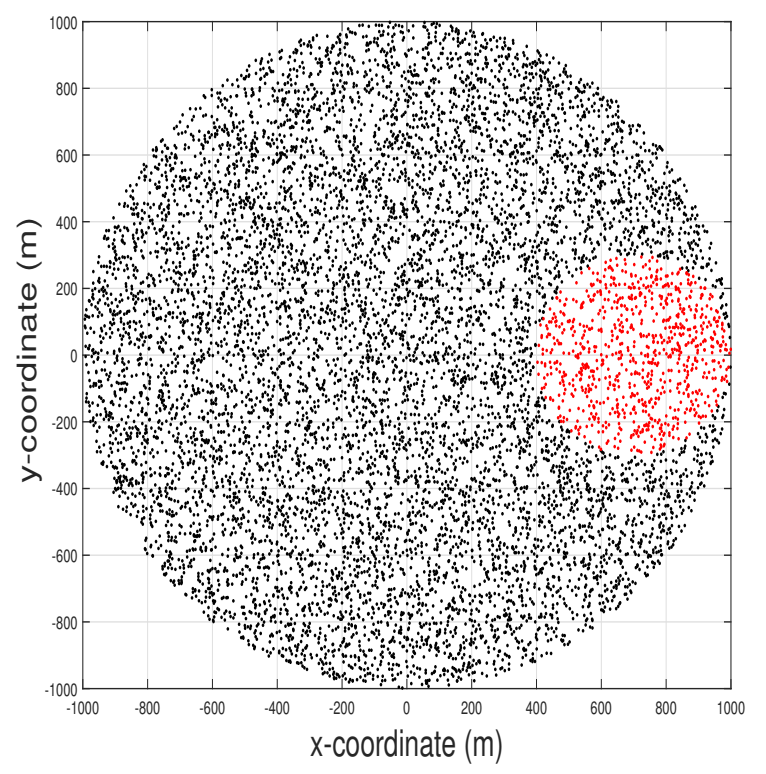

Fig. 11. Coverage areas of the MBS and PBS when the MBS and PBS are located at $(0,0)$ and $(566,0)$, respectively.

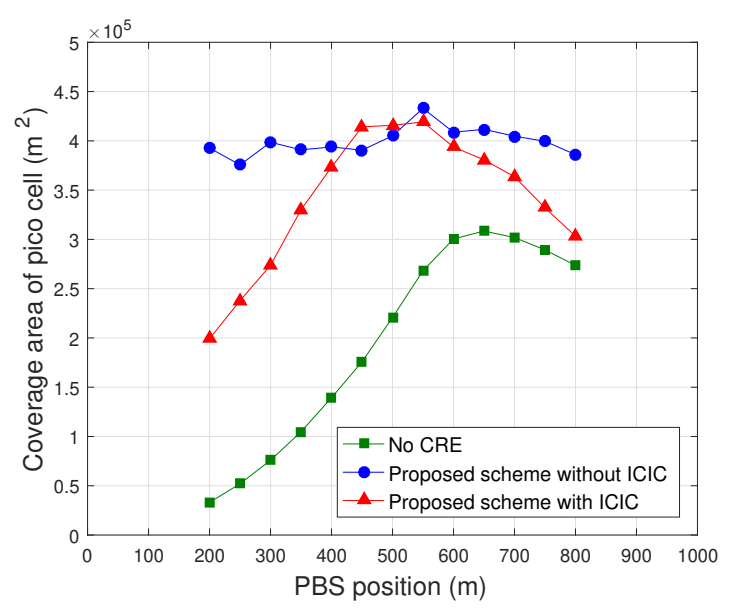

Fig. 12. Coverage area of the PBS with no CRE, with the proposed CRE only, and with the proposed CRE plus ICIC in the 1-PBS model.

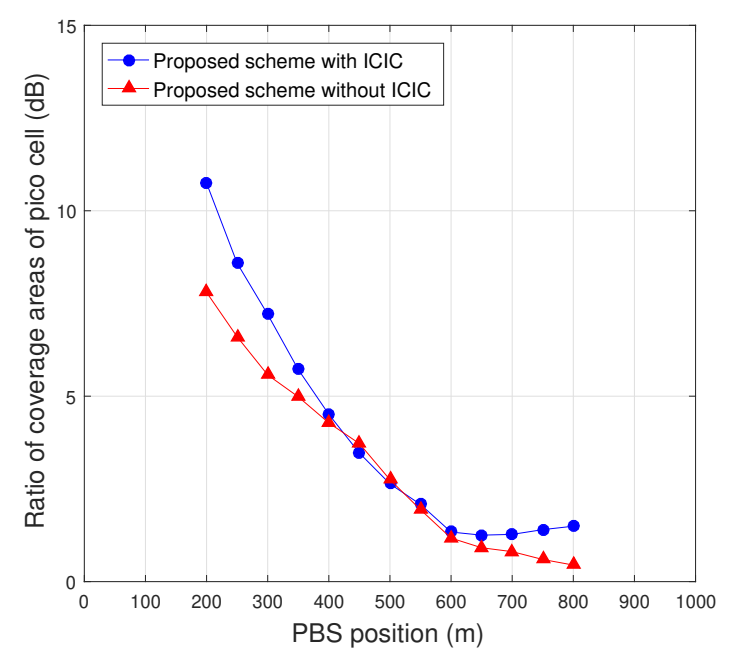

Fig. 13. Ratio of the coverage area with the proposed CRE to the coverage area without CRE in the 1-PBS model.

\subsection{Offloading Performance}

Figures 14 and 15 show the offloading performance of the proposed CRE scheme with optimum bias. It is clearly observed that the number of users in the pico-cell increases when the proposed CRE scheme is applied. Note also that the offloading performance (in terms of the ratio of the number of users in the pico-cell to the total number of users) with the proposed CRE scheme plus the ICIC is less sensitive to the distance between the MBS and PBS: $2 \%-10 \%$ versus $12 \%-14 \%$ and $3 \%-29 \%$ versus $17 \%-29 \%$, in the $1-$ and 3-PBS models, respectively. In addition, it is confirmed that the ICIC in the proposed CRE scheme provides more balance in the loading performance: A possible observation is that the ICIC in the proposed CRE scheme reduces the influence of the distance between the MBS and PBS on the number of users in the pico-cell.

Figures 16 and 17 show the average rates of users in the 1- and 3-PBS models, respectively. Here, 'Macro' and 'Pico' in the legends represents average rate of a user in the macro- and pico-cells, respectively. From the results shown in these two figures we can make the following observations: (A) The average rates of users in the macro-cell do not change considerably with the proposed CRE scheme. (B) The variation, as the distance between the MBS and PBS changes, of the average rates of users in the pico-cell with the proposed CRE scheme is smaller than that without the proposed CRE scheme. (C) If the ICIC is employed in addition to the proposed CRE scheme, the average rates of users in the pico-cell increase significantly. In passing, let us note that, since the average rate of pico-cell users is much higher than that of macro-cell users, macro-cell users may complain about such unfairness: Finding a fairer CRE scheme would be an interesting research topic.

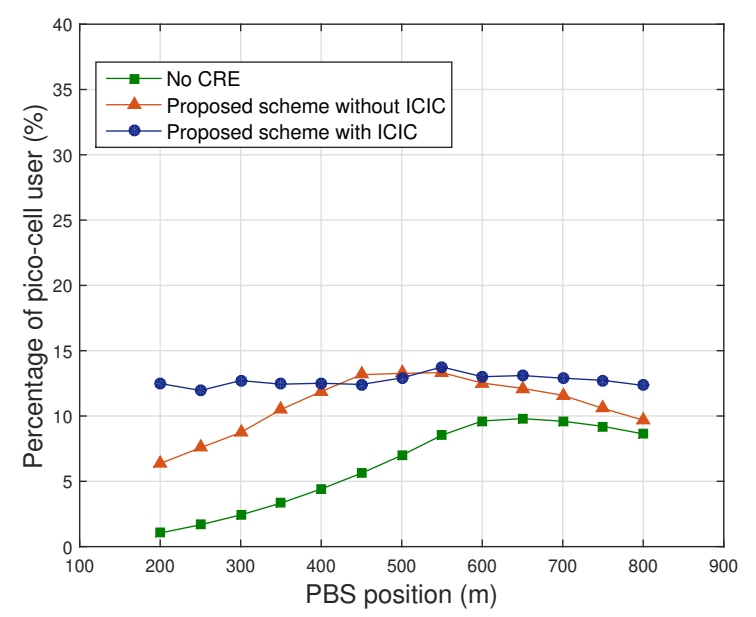

Fig. 14. Offloading performance of the proposed CRE scheme with optimum bias in the 1-PBS model. 


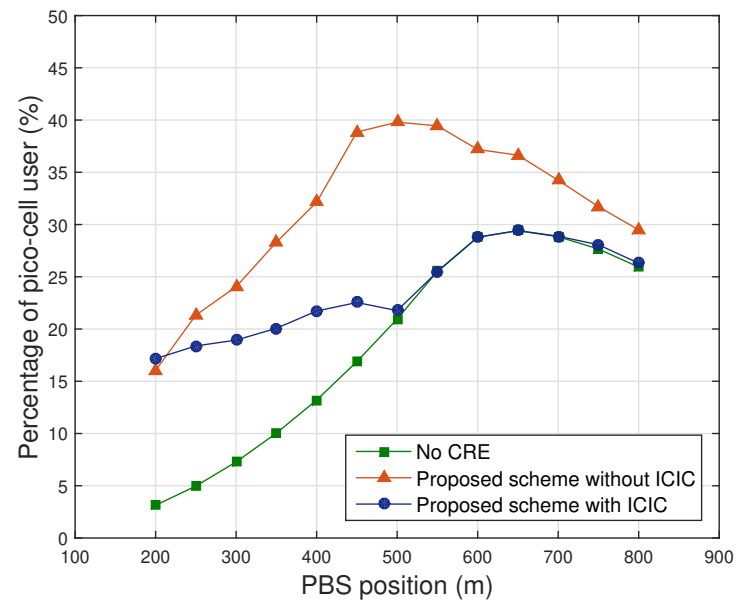

Fig. 15. Offloading performance of the proposed CRE scheme with optimum bias in the 3-PBS model.

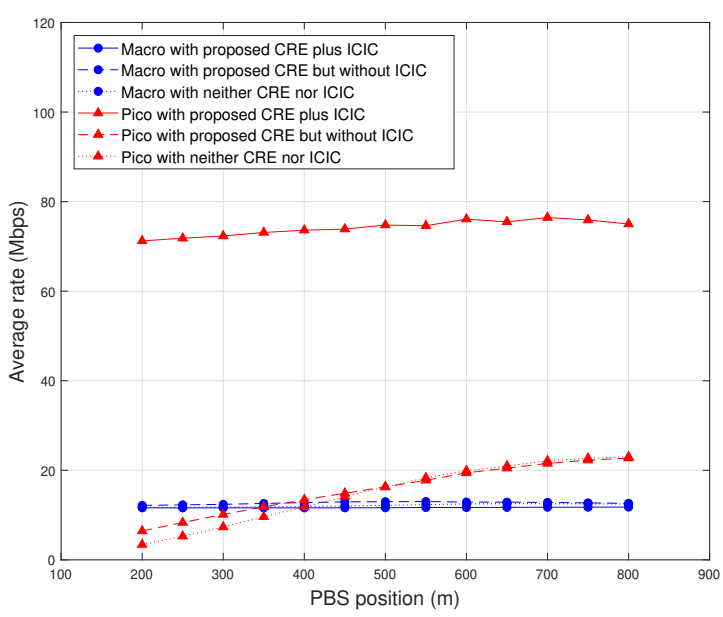

Fig. 16. Average rate of users in the 1-PBS model.

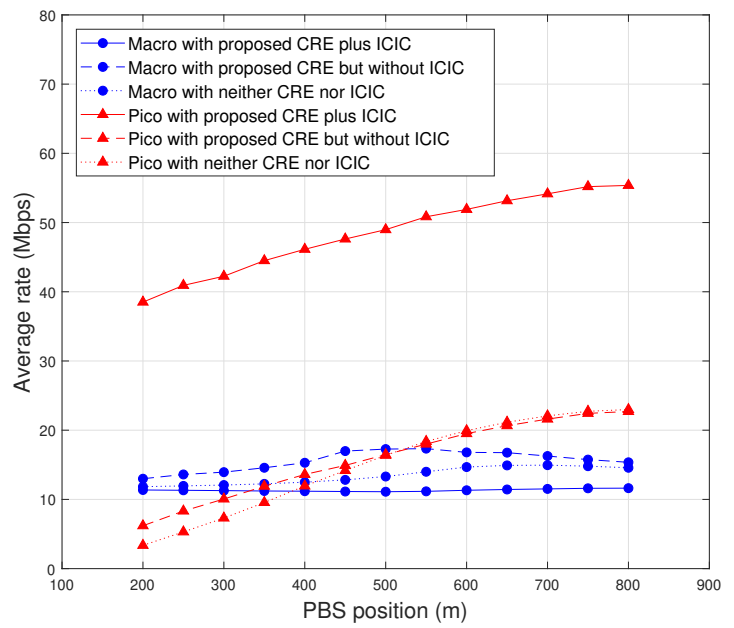

Fig. 17. Average rate of users in the 3-PBS model.
We would like to mention that the goal in this paper is to maximize the overall sum rate in the macro- and pico-cells, because of which the basic purpose (providing balance in the loading of the MBS and PBS while requiring some users intentionally to operate at lower SINR, or equivalently, increasing the capacity of the system) of CRE may be achieved only partially. It should also be noted that, while some users (those in the macro-cell after the CRE) can enjoy more favorable environment with the proposed CRE scheme, some of the other users (especially those originally in the pico-cell) may have to operate at lower SINR after the proposed CRE scheme is employed since the number of users in the picocell will tend to be larger than that before the CRE scheme is employed. This is common to all CRE schemes.

\section{Concluding Remark}

Heterogeneous networks have recently been proposed as an attractive solution to overcome the deficiency of invaluable spectrum resource. The scheme of CRE has been reported to be successful in overcoming the drawback of heterogeneous network and intensifying the merit of heterogeneous network. In heterogeneous networks, we have addressed in this paper a novel CRE scheme, in which the positions of pico base stations are taken into account for the determination of the bias. The proposed CRE scheme is shown to perform better when it is employed together with an inter-cell interference coordination scheme.

The optimal bias of the proposed CRE scheme is observed to depend on the relative position of PBSs to the MBS. We have presented simulation results in one-PBS and threePBS models and confirmed that the sum rate is increased with the proposed CRE scheme. It is noteworthy that the optimal bias tends to decrease when PBSs are located farther from the MBS. In addition, the proposed CRE scheme with the optimal bias is shown to provide higher sum rate and more balanced load among base stations. Finding a sub-optimal value of the bias via a simpler method would be an interesting topic to pursue.

\section{Acknowledgments}

This work was supported by the National Research Foundation of Korea under Grant NRF-2018R1A2A1A05023192 with funding from the Ministry of Science and Information and Communication Technology, for which the authors wish to express their appreciation. The authors would also like to thank the Associate Editor and two anonymous reviewers for their constructive suggestions and helpful comments. 


\section{References}

[1] ANDREWS, J. G. Seven ways that HetNets are a cellular paradigm shift. IEEE Communications Magazine, 2013, vol. 51, no. 3, p. 136-144. DOI: 10.1109/MCOM.2013.6476878

[2] BOGALE, T. E., LE, L. B. Massive MIMO and mm wave for $5 \mathrm{G}$ wireless HetNet. IEEE Vehicular Technology Magazine, 2016, vol. 11, no. 1, p. 64-75. DOI: 10.1109/MVT.2015.2496240

[3] KLEIN, T. E., HAN, S.-J. Assignment strategies for mobile data users in hierarchical overlay networks: Performance of optimal and adaptive strategies. IEEE Journal on Selected Areas in Communications, 2004, vol. 22, no. 5, p. 849-861. DOI: 10.1109/JSAC.2004.826922

[4] FOOLADIVANDA, D., ROSENBERG, C. Joint resource allocation and user association for heterogeneous wireless cellular networks. IEEE Transactions on Wireless Communications, 2013, vol. 12, no. 1, p. 248-257. DOI: 10.1109/TWC.2012.121112.120018

[5] CHANDRASEKHAR, V., ANDREWS, J. G., MUHAREMOVIC, T., et al. Power control in two-tier femtocell networks. IEEE Transactions on Wireless Communications, 2009, vol. 8, no. 8, p. 4316-4328. DOI: 10.1109/TWC.2009.081386

[6] CHANDRASEKHAR, V., KOUNTOURIS, M., ANDREWS, J. G. Coverage in multi-antenna two-tier networks. IEEE Transactions on Wireless Communications, 2009, vol. 8, no. 10, p. 5314-5327. DOI: 10.1109/TWC.2009.090241

[7] LOPEZ-PEREZ, D., CHU, X., GUVENC, I. On the expanded region of picocells in heterogeneous networks. IEEE Transactions on Wireless Communications, 2012, vol. 6, no. 3, p. 281-294. DOI: $10.1109 /$ JSTSP.2012.2190381

[8] CHO, S., CHOI, W. Coverage and load balancing in heterogeneous cellular networks with minimum cell separation. IEEE Transactions on Mobile Computing, 2014, vol. 13, no. 9, p. 1955-1966. DOI: 10.1109/TMC.2013.87

[9] DI RENZO, M., GUIDOTTI, A., G. CORAZZA, E. Average rate of downlink heterogeneous cellular networks over generalized fading channels: A stochastic geometry approach. IEEE Transactions on Communications, 2013, vol. 61, no. 7, p. 3050-3071. DOI: 10.1109/TCOMM.2013.050813.120883

[10] NIGAM, G., MINERO, P., HAENGGI, M. Coordinated multipoint joint transmission in heterogeneous networks. IEEE Transactions on Communications, 2014, vol. 62, no. 11, p. 4134-4146. DOI: 10.1109/TCOMM.2014.2363660

[11] SINGH, S., ANDREWS, J. G. Joint resource partitioning and offloading in heterogeneous cellular networks. IEEE Transactions on Wireless Communications, 2014, vol. 13, no. 2, p. 888-901. DOI: 10.1109/TWC.2013.120713.130548

[12] MADAN, R., BORRAN, J., SAMPATH, A., et al. Cell association and interference coordination in heterogeneous LTE-A cellular networks. IEEE Journal on Selected Areas in Communications, 2010, vol. 28, no. 9, p. 1479-1489. DOI: 10.1109/JSAC.2010.101209

[13] GUVERNC, I. Capacity and fairness analysis of heterogeneous networks with range expansion and interference coordination. IEEE Communications Letters, 2011, vol. 15, no. 10, p. 1084-1087. DOI: 10.1109/LCOMM.2011.082611.111387

[14] OH, J., HAN, Y. Cell selection for range expansion with almost blank subframe in heterogeneous networks. In Proceedings of the IEEE Annual International Symposium on Personal, Indoor, and Mobile Radio Communications. Sydney (Australia), 2012, p. 653-657. DOI: 10.1109/PIMRC.2012.6362865
[15] KIKUCHI, K., OTSUKA, H. Proposal of adaptive control CRE in heterogeneous networks. In Proceedings of the IEEE Annual International Symposium on Personal, Indoor, and Mobile Radio Communications. Sydney (Australia), 2012, p. 910-914. DOI: 10.1109/PIMRC.2012.6362914

[16] YE, Q., RONG, B., CHEN, Y., et al. User association for load balancing in heterogeneous cellular networks. IEEE Transactions on Wireless Communications, 2013, vol. 12, no. 6, p. 2706-2716. DOI: 10.1109/TWC.2013.040413.120676

[17] TANG, H., PENG, J., HONG, P., et al. Offloading performance of range expansion in picocell networks: A stochastic geometry analysis. IEEE Wireless Communications Letters, 2013, vol. 2, no. 5, p. 511-514. DOI: 10.1109/WCL.2013.061913.130346

[18] KUDO, T., OHTSUKI, T. Cell range expansion using distributed Q-learning in heterogeneous networks. EURASIP Journal on Wireless Communications and Networking, 2013, vol. 1, article 61, 10 pages. DOI: 10.1186/1687-1499-2013-61

[19] KIKUCHI, K., OTSUKA, H. Parameter optimization for adaptive control CRE in HetNet. In Proceedings of the IEEE Annual International Symposium on Personal, Indoor, and Mobile Radio Communications. London (UK), 2013, p. 3334-3338. DOI: 10.1109/PIMRC.2013.6666723

[20] DENG, X., WANG, Y., GU, X., et al. Analysis of cell range extension and a bias configuration strategy in dense small cell networks. In Proceedings of the International Conference on Network Infrastructure and Digital Content. Beijing (China), 2014, p. 425-429. DOI: 10.1109/ICNIDC.2014.7000338

[21] GU, X., LI, W., ZHANG, L. Adaptive cell range control in heterogeneous network. In Proceedings of the International Conference on Wireless Communications and Signal Processing. Hangzhou (China), 2013, p. 1-5. DOI: 10.1109/WCSP.2013.6677262

[22] GU, X., DENG, X., LI, Q., et al. Capacity analysis and optimization in heterogeneous network with adaptive cell range control. International Journal of Antennas and Propagation, 2014, vol. 2014, Article ID 215803, 10 pages. DOI: 10.1155/2014/215803

[23] JINDAL, N., ANDREWS, J. G., WEBER, S. Multi-antenna communication in ad hoc networks: Achieving MIMO gains with SIMO transmission. IEEE Transactions on Communications, 2011, vol. 59, no. 2, p. 529-540. DOI: 10.1109/TCOMM.2010.120710.090793

[24] 3GPP, TR 36.814 (V9.0.0), Further Advancements for E-UTRA Physical Layer Aspects, 2010.

[25] CADAMBE, V. R., JAFAR, S. A. Interference alignment and degrees of freedom of the k-user interference channel. IEEE Transactions on Information Theory, 2008, vol. 54, no. 8, p. 3425-3441. DOI: 10.1109/TIT.2008.926344

[26] GOMADAM, K., CADAMBE, V. R., JAFAR, S. A. A distributed numerical approach to interference alignment and applications to wireless interference networks. IEEE Transactions on Information Theory, 2011, vol. 57, no. 6, p. 3309-3322. DOI: 10.1109/TIT.2011.2142270

\section{About the Authors ...}

Taesung JUNG received the B.S. and M.S. degrees in Electrical Engineering from Korea Advanced Institute of Science and Technology, Daejeon, Korea, in 2015 and 2017, respectively. His research interests include wireless communication, signal processing and heterogeneous network. 
Iickho SONG received the B.S.E. (magna cum laude) and M.S.E. degrees in Electronics Engineering from Seoul National University, Seoul, Korea, in 1982 and 1984, respectively, and the M.S.E. and Ph.D. degrees in Electrical Engineering from the University of Pennsylvania, Philadelphia, PA, USA, in 1985 and 1987, respectively. He was a Member of the Technical Staff at Bell Communications Research in 1987. In 1988, he joined the School of Electrical Engineering, Korea Advanced Institute of Science and Technology, Daejeon, Korea, where he is currently a Professor. He has coauthored a few books including Advanced Theory of Signal Detection (Springer, 2002) and Random Variables and Random Processes (in Korean; Freedom Academy, 2014), and published papers on signal detection and mobile communications. Prof. Song is a Fellow of the Korean Academy of Science and Technology (KAST). He is also a Fellow of the IEEE and IET, and a Member of the Acoustical Society of Korea (ASK), Institute of Electronics Engineers of Korea (IEEK), Korean Institute of Communications and Information Sciences (KICS), and Korea Institute of Information, Electronics, and Communication Technology (KIIECT). He has served as the Treasurer of the IEEE Korea Section, an Editor of the Journal of the ASK, an Editor of Journal of the IEEK, an Editor of the Journal of the KICS, an Editor of the Journal of Communications and Networks (JCN), and a Division Editor of the $J C N$. He has received several awards including the Young Scientists Award (KAST, 2000), Achievement Award (IET, 2006), and Hae Dong Information and Communications Academic Award (KICS, 2006).

Seungwon LEE received the B.S.E. degree in Electronics Engineering from Kyung Hee University, Yongin, Korea, in 2010, and the M.S.E. degree in Electrical Engineering from Korea Advanced Institute of Science and Technology (KAIST), Daejeon, Korea, in 2012. He is currently working toward the Ph.D. degree at KAIST. Since February 2010, he has been a Teaching and Research Assistant in the School of Electrical Engineering, KAIST. His research interests include mobile communications, detection and estimation theory, and statistical signal processing.

Seungjae JUNG received the B.S. degree in Electronics and Electrical Engineering from Sungkyunkwan University, Suwon, Korea, in 2013 and the M.S. degree in Electrical Engineering from Korea Advanced Institute of Science and Technology (KAIST), Daejeon, Korea, in 2015. He is cur- rently pursuing the Ph.D. degree in Electrical Engineering at KAIST. His research interests include wireless communication, physical-layer secrecy, energy harvesting, signal processing for communication, and information theory.

Seokho YOON (corresponding author) received the B.S.E. (summa cum laude), M.S.E., and Ph.D. degrees in Electrical Engineering from Korea Advanced Institute of Science and Technology (KAIST), Daejeon, Korea, in 1997, 1999, and 2002, respectively. From March 2002 to June 2002, he was with the Department of Electrical Engineering and Computer Sciences, Massachusetts Institute of Technology (MIT), Cambridge, MA, and from July 2002 to February 2003, he was with the Department of Electrical Engineering, Harvard University, Cambridge, MA, as a Postdoctoral Research Fellow. In March 2003, he joined the College of Information and Communication Engineering, Sungkyunkwan University, Suwon, Korea, where he is currently a Professor. His research interests include spread spectrum and OFDM systems, mobile/satellite communications, detection and estimation theory, and statistical signal processing. Dr. Yoon is a Senior Member of the Institute of Electrical and Electronics Engineers (IEEE), a Member of the Institute of Electronics, Information and Communication Engineers (IEICE), and a Lifetime Member of the Korean Institute of Communication Sciences (KICS).

Joonhyuk KANG received his B.S.E. and M.S.E. degrees from Seoul National University, Seoul, Korea, 1991 and 1993, respectively. He got his Ph. D. degree in Telecommunication and Information System Engineering at the University of Texas at Austin in 2002. He is currently working as a faculty member of Korea Advanced Institute of Science and Technology. He spent five years as an R\&D engineer at Samsung Electronics, where he was involved in R\&D for DSP-based real-time control systems. He also spent eight months at $\mathrm{Cwill}$ Telecommunications, U.S.A., where he worked on multi-carrier CDMA system with antenna array. His research interests include cognitive radio, cooperative communications, MIMO-OFDM wireless system and location based service (LBS) application. He is a member of the Institute of Electrical and Electronics Engineers (IEEE), Institute of Electronics, Information and Communication Engineers (IEICE), and Tau Beta Pi, and a recipient of TxTEC Fellowship, 2000-2002. 